\title{
BMJ Open Effect of ephedrine and phenylephrine on brain oxygenation and microcirculation in anaesthetised patients with cerebral tumours: study protocol for a randomised controlled trial
}

Klaus Ulrik Koch, ${ }^{1}$ Anna Tietze, ${ }^{2,3}$ Joel Aanerud, ${ }^{4}$ Gorm von Öettingen, ${ }^{5}$ Niels Juul, ${ }^{1}$ Jens Christian Hedemann Sørensen, ${ }^{5}$ Lone Nikolajsen, ${ }^{1}$ Leif Østergaard, ${ }^{2}$ Mads Rasmussen ${ }^{1}$

To cite: Koch KU, Tietze A, Aanerud $\mathrm{J}$, et al. Effect of ephedrine and phenylephrine on brain oxygenation and microcirculation in anaesthetised patients with cerebral tumours: study protocol for a randomised controlled trial. BMJ Open 2017;7:e018560. doi:10.1136/ bmjopen-2017-018560

- Prepublication history and additional material for this paper are available online. To view these files, please visit the journal online (http://dx.doi. org/10.1136/bmjopen-2017018560).

Received 6 July 2017

Revised 9 October 2017

Accepted 17 October 2017

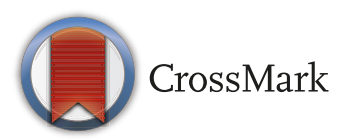

For numbered affiliations see end of article.

Correspondence to

Dr Klaus Ulrik Koch;

klaukoch@rm.dk

\begin{abstract}
Introduction During brain tumour surgery, vasopressor drugs are commonly administered to increase mean arterial blood pressure with the aim of maintaining sufficient cerebral perfusion pressure. Studies of the commonly used vasopressors show that brain oxygen saturation is reduced after phenylephrine administration, but unaltered by ephedrine administration. These findings may be explained by different effects of phenylephrine and ephedrine on the cerebral microcirculation, in particular the capillary transit-time heterogeneity, which determines oxygen extraction efficacy. We hypothesised that phenylephrine is associated with an increase in capillary transit-time heterogeneity and a reduction in cerebral metabolic rate of oxygen compared with ephedrine. Using MRI and positron emission tomography (PET) as measurements in anaesthetised patients with brain tumours, this study will examine whether phenylephrine administration elevates capillary transit-time heterogeneity more than ephedrine, thereby reducing brain oxygenation.
\end{abstract}

Methods and analysis This is a double-blind, randomised clinical trial including 48 patients scheduled for surgical brain tumour removal. Prior to imaging and surgery, anaesthetised patients will be randomised to receive either phenylephrine or ephedrine infusion until mean arterial blood pressure increases to above $60 \mathrm{~mm} \mathrm{Hg}$ or $20 \%$ above baseline. Twenty-four patients were allocated to MRI and another 24 patients to PET examination. MRI measurements include cerebral blood flow, capillary transit-time heterogeneity, cerebral blood volume, blood mean transit time, and calculated oxygen extraction fraction and cerebral metabolic rate of oxygen for negligible tissue oxygen extraction. PET measurements include cerebral metabolic rate of oxygen, cerebral blood flow and oxygen extraction fraction. Surgery is initiated after MRI/PET measurements and subdural intracranial pressure is measured.

Ethics and dissemination This study was approved by the Central Denmark Region Committee on Health
Strengths and limitations of this study

- Unique clinical trial design incorporating neuroimaging in the search for optimal vasopressors in anaesthetised patients undergoing craniotomy for cerebral tumours.

- Comparing effects of vasopressors on cerebral blood flow parameters between the peritumourous area and the contralateral hemisphere.

- Neuroimaging with MRI and positron emission tomography (PET) in a homogeneous group of patients to obtain the best measurements of cerebral blood flow, capillary transit-time heterogeneity, cerebral metabolic rate of oxygen and oxygen extraction fraction.

- Small sample size due to difficult inclusion in a vulnerable group of patients and extensive study set-up.

- Not possible to obtain simultaneous MRI and PET values; thus, the study is divided into two parts.

Research Ethics (12 June 2015; 1-10-72-116-15). Results will be disseminated via peer-reviewed publication and presentation at international conferences.

Trial registration number NCT02713087; Pre-results. 2015-001359-60; Pre-results.

\section{INTRODUCTION}

Patients with brain tumours often have increased intracranial pressure $(\text { ICP })^{1}$ and impaired autoregulation, ${ }^{2}$ with potential to cause cerebral ischaemia during surgery. ${ }^{34}$ To ensure sufficient cerebral perfusion pressure (CPP), higher mean arterial blood pressure (MABP) values ${ }^{56}$ are often maintained with vasopressor therapy in patients undergoing 
craniotomy for brain tumours and other cerebral pathology. $^{7}$

Phenylephrine and ephedrine are commonly used vasopressors during neurosurgical procedures. ${ }^{8}{ }^{9}$ However, studies show that MABP correlates poorly with microcirculatory flow. ${ }^{10-12}$ Accordingly, elevation of blood pressure may, paradoxically, lead to reduced capillary perfusion and decreased oxygen delivery despite reaching endpoints with CPP within $50-70 \mathrm{~mm} \mathrm{Hg}{ }^{10-12}$ This is supported by an experimental study demonstrating a vasopressor-inotrope-induced mismatch between cerebral perfusion and oxygenation, possibly due to microvascular heterogeneity. ${ }^{13}$ In addition, a number of studies demonstrate that cerebral tissue oxygen saturation $\left(\mathrm{SctO}_{2}\right)$, as measured by near-infrared spectroscopy (NIRS), decreases after phenylephrine administration but remains unchanged after administration of ephedrine, although both agents cause MABP to increase in healthy anaesthetised subjects. ${ }^{9} 14-18$ In one study, the decrease in $\mathrm{SctO}_{2}$ was associated with an increase in cerebral artery blood flow velocity and possibly vasoconstriction. ${ }^{19}$ Thus, augmenting MABP and CPP with vasopressors may lead to a paradoxical microcirculatory response, including vasopressor-induced tissue hypoperfusion and poor tissue oxygenation. Except for one study reporting transcranial Doppler, cerebral blood flow (CBF) was not measured in the studies above, and it is therefore unclear whether the $\mathrm{SctO}_{2}$ changes were associated with changes in $\mathrm{CBF}^{15}$

Brain microcirculation is the primary site of oxygen exchange. We have recently proposed that red blood cell capillary transit-time heterogeneity (CTH) may affect tissue oxygen tension in patients with ischaemic stroke, subarachnoid haemorrhage and traumatic brain injury. ${ }^{20-23}$

According to this theory, capillary constriction, or capillary compression due to oedema and elevated ICP, may cause redistribution of capillary flows into heterogeneous patterns with functional shunting of oxygenated blood through the capillary bed. ${ }^{20}$ This capillary dysfunction may further hinder oxygen diffusion into cerebral tissue and ultimately cause cerebral ischaemic damage. We therefore hypothesise that the diverging effects of phenylephrine and ephedrine on cerebral oxygenation may be the result of differential effects on either blood supply, as measured by $\mathrm{CBF}$, or oxygen extraction efficacy (OEF), as derived from $\mathrm{CTH}$.

Currently, there are no comparative studies available on the effects of commonly used vasopressors on brain microcirculation and oxygenation in patients with brain pathology. Using MRI and positron emission tomography (PET), this study aims to investigate whether phenylephrine, when compared with ephedrine, is associated with capillary flow disturbances, elevated CTH and reduced oxygen availability in anaesthetised patients with brain tumours.
METHODS AND ANALYSIS

\section{Study design}

The study is an investigator-initiated, interventional, prospective and randomised clinical trial. The setting for the trial is the Department of Neurosurgery and Neuroanesthesia, Aarhus University Hospital, Aarhus, Denmark. The trial is divided into two parts according to imaging method (see below). Trial reporting will be guided by the Standard Protocol Items: Recommendations for Interventional Trials (SPIRIT) guidelines (see online supplementary file 1 and file 2 for the populated SPIRIT checklist and figure).

\section{Patients}

Forty-eight patients scheduled for intracranial tumour resection by craniotomy will be included in the study following written informed consent obtained by the primary investigator or coinvestigator.

\section{Inclusion criteria}

Patients who meet the following criteria will be included in the study: (1) patients who are scheduled for elective craniotomy for supratentorial malignant and non-malignant brain tumours $3 \mathrm{~cm}$ or larger (measured as the largest diameter in any plane on MRI), (2) patients with an American Society of Anesthesiologists status $1-3,{ }^{24}$ and (3) patients aged between 18 and 75 years.

\section{Exclusion criteria}

Patients who meet any of the following criteria will be excluded from the study: (1) patients who are pregnant (positive pregnancy urine test) or nursing, (2) patients with a history of allergic reactions to phenylephrine or ephedrine, (3) patients with an extra glomerular filtration ratio (eGFR) $<60 \mathrm{~mL} / \mathrm{min}$ per $1.73 \mathrm{~m}^{2}$ and (4) and patients who are unable to give written informed consent.

\section{Randomisation and blinding}

Patients will be allocated to treatment groups based on a computer-generated random allocation sequence (http://www.randomization.com), using a 1:1 randomisation ratio. In parts 1 and 2 of the study, randomisation is performed in blocks of $10+10+4$ patients to receive either phenylephrine or ephedrine. The allocation sequence refers an independent assistant to one of the consecutively numbered, opaque, sealed envelopes, which contains information about the vasopressor to be given (phenylephrine or ephedrine). On study inclusion, each patient receives the treatment assigned in a numbered envelope, which will be opened by dedicated study nurses just before anaesthesia. Syringes containing either ephedrine or phenylephrine are marked with a randomisation code known only to the study nurses.

Patients and investigators are blinded to the randomisation. Interpretation of MR/PET imaging and calculation of flow parameters are performed by a blinded investigator. After final inclusion, an independent assistant will unblind the two groups and subsequently label the 
groups 0 and 1 . Statistical analysis will be performed and eventually final unblinding will take place.

\section{Anaesthesia and monitoring}

Anaesthesia is induced with propofol $0.7-1.5 \mathrm{mg} / \mathrm{kg}$ and remifentanil $0.3-0.6 \mathrm{~mL} / \mathrm{kg} / \mathrm{h}$. A low dose of suxamethonium is administered in order to facilitate intubation. This is in order to reduce risk of severe hypotension after high induction doses of propofol and remifentanil. Nondepolarising relaxation is not used. It is important to emphasise that bispectral index (BIS) monitoring is used during induction to ensure adequate depth of anaesthesia. ${ }^{9}$ Propofol and remifentanil are administered to ensure immobility. The patients are ventilated with $50 \%$ oxygen in air by controlled ventilation with the goal of achieving partial pressure of carbon dioxide in arterial blood $\left(\mathrm{PaCO}_{2}\right)$ and partial pressure of oxygen in arterial blood $\left(\mathrm{PaO}_{2}\right)$ values between 30 and $40 \mathrm{~mm} \mathrm{Hg}$ and greater than $100 \mathrm{~mm} \mathrm{Hg}$, respectively. $\mathrm{PaCO}_{2}$ is adjusted according to arterial blood gas measurement. Isotonic $\mathrm{NaCl}$ or Ringer's lactate is infused at a rate of $3 \mathrm{~mL} / \mathrm{kg} /$ hour. Temperature is monitored as soon as the patient arrives in the operating room-equipment for temperature measurement is not MRI-compatible. The patient is kept warm in the scanner with sheets and blankets; furthermore, the MRI increases temperature in the patient body during the scan. The patient is supine and neck rotation and compression cannot occur in our MRI head cradles.

ECG, oxygen saturation, heart rate and intra-arterial blood pressure are continuously monitored. Depth of anaesthesia is monitored with BIS. Brain oxygenation is monitored by NIRS before and after MRI, and during PET, to allow comparison of brain oxygenation measurements determined by PET and MRI, respectively. Subdural ICP is measured perioperatively after removal of the bone flap. ${ }^{25}$

\section{Intervention and study drugs}

Participants will receive an intravenous infusion of either ephedrine $(2 \mathrm{mg} / \mathrm{mL})$ or phenylephrine $(0.1 \mathrm{mg} / \mathrm{mL})$ until MABP increases to above $60 \mathrm{~mm} \mathrm{Hg}$, or by $20 \%$ relative to the baseline. Baseline MABP is defined as the first MABP measured at the time of the initial MRI or PET sequence. The infusion is terminated after the subdural ICP measurement. These infusion protocols were chosen to reflect clinical recommendations and aimed to maintain a stable blood pressure throughout the whole MRI and PET sequences. ${ }^{26}{ }^{27}$ In case of unacceptable hypertension during infusion of study drug, it was considered to reduce or end infusion. In addition, if reflex bradycardia occurs we consider to reduce study drug infusion rate or administration of atropine.

\section{Experimental MRI and PET protocol}

The experimental protocol is shown in figure 1. For logistical reasons, and to avoid long duration of anaesthesia, we choose not to perform consecutive MRI and PET on the same patient. Instead the protocol consists of two parts, according to imaging method.

- Part 1 (MRI study): 24 patients are randomised to infusion of either ephedrine or phenylephrine and imaged by MRI after induction of anaesthesia. ${ }^{14}$

- Part 2 (PET study): 24 patients are randomised to infusion of either ephedrine or phenylephrine and imaged by PET after induction of anaesthesia. ${ }^{14}$

The overall experimental set-up is the same for the two parts of the study. The patient is anaesthetised in a room adjacent to the MRI or PET scanner. MRI and PET examinations are performed before and after administration of the study drug. The study period is shown in figure 2. The specific MRI and PET protocols are described below.

\section{MRI protocol}

The protocol consists of the following: (1) conventional MRI sequences to depict brain anatomy and structural changes, (2) diffusion-weighted MRI including diffusion kurtosis imaging to infer tissue microstructure,,$^{28}$ and (3) perfusion-weighted MRI to infer cerebral haemodynamics. The duration of the

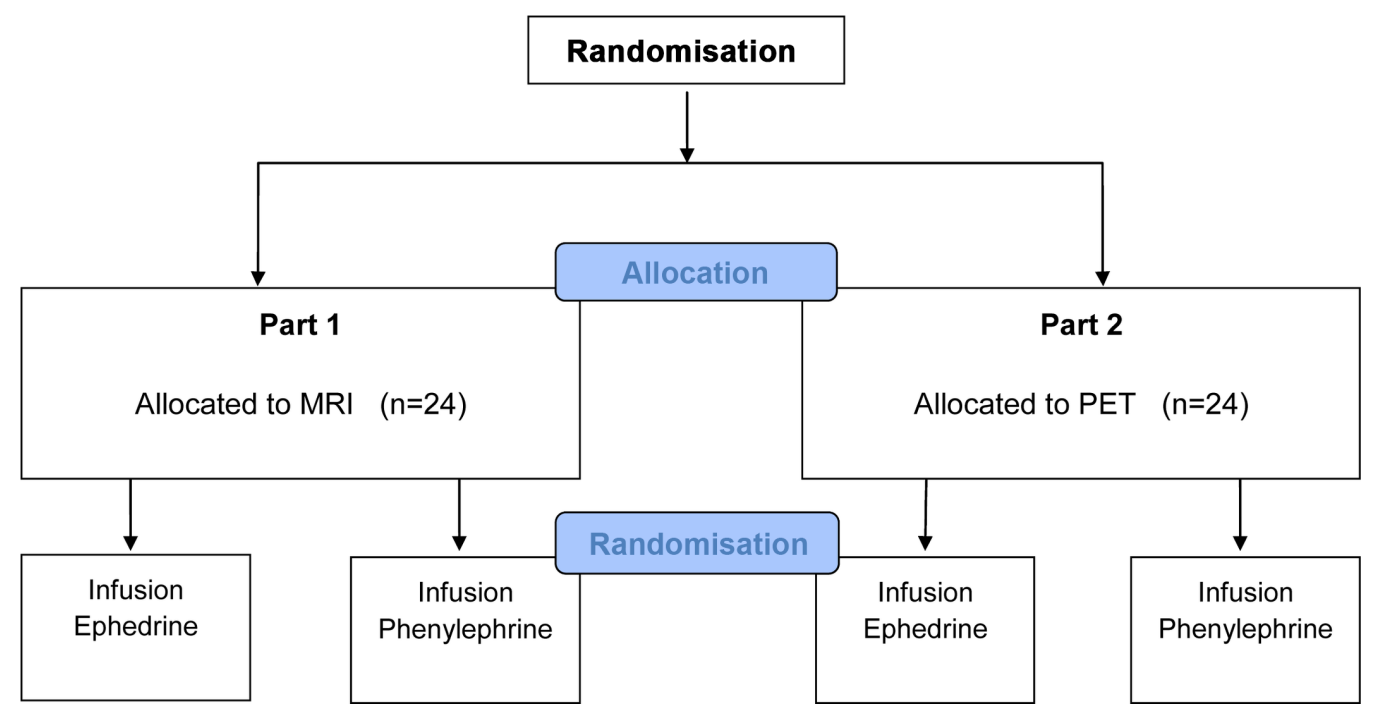

Figure 1 Study flow chart. PET, positron emission tomography. 


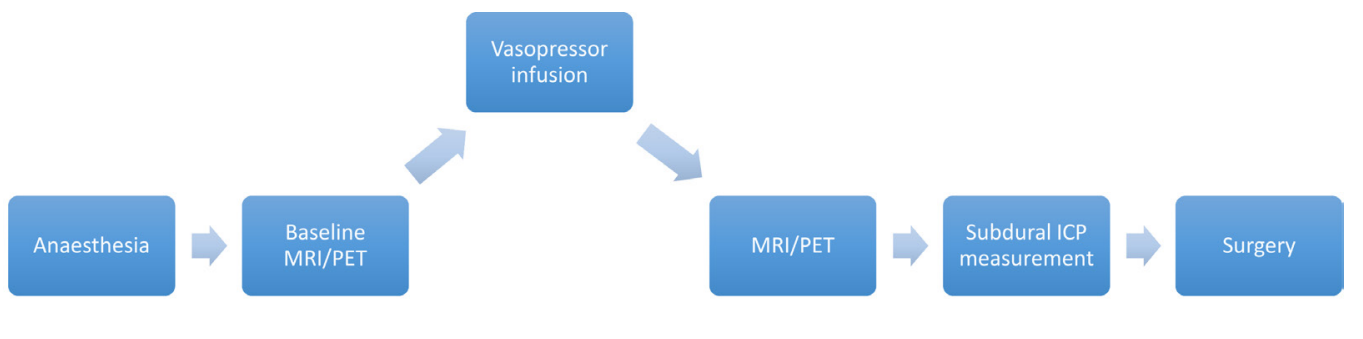

Figure 2 Time sequence diagram. Anaesthesia induced at time $0 \mathrm{~min}$. During the first hour baseline MRI/positron emission tomography (PET) is performed (time approximately $60 \mathrm{~min}$ ). Vasopressor infusion of either phenylephrine or ephedrine is initiated until mean arterial blood pressure (MABP) increases to above $60 \mathrm{~mm} \mathrm{Hg}$, or by $20 \%$ relative to the baseline. A $5 \mathrm{~min}$ steady-state period of MABP is maintained before the second MRI/PET examination is performed (time approximately $100 \mathrm{~min}$ ). The patient is transported to the operating room. Surgery is initiated, and after removal of the bone flap subdural intracranial pressure (ICP) is measured (time approximately $140 \mathrm{~min}$ ). Subsequently the study period is terminated and surgery continues (time approximately $150 \mathrm{~min}$ ).

entire MRI scan is estimated at $60 \mathrm{~min}$, and $0.25 \mathrm{mmol} / \mathrm{kg}$ macrocyclic gadolinium MRI contrast is administered, corresponding to 2.5 standard doses.

\section{PET protocol}

The protocol consists of the following: (1) CBF: $500 \mathrm{MBq}$ of $\left[{ }^{15} \mathrm{O}\right] \mathrm{H}_{2} \mathrm{O}$ is injected intravenously. On injection, a $5 \mathrm{~min}$ PET image acquisition of the brain is started together with arterial blood sampling (also $5 \mathrm{~min}$ ) with an automated blood radioactivity sampler that draws $7 \mathrm{~mL}$ blood per minute. The PET acquisition is performed on a Siemens High Resolution Research Tomograph in list mode. (2) Cerebral metabolic rate of oxygen $\left(\mathrm{CMRO}_{2}\right): 1000 \mathrm{MBq}$ of $\left[{ }^{15} \mathrm{O}\right] \mathrm{O}_{2}$ is inhaled and immediately exhaled. On inhalation, a $3 \mathrm{~min}$ PET acquisition and blood sampling are performed with protocols identical to that of the CBF measurement. (3) During each scan session, oxygen saturation and haemoglobin concentration will be measured.

\section{Endpoints}

Primary endpoint

- Between-groups difference in CTH measured with MRI.

\section{Secondary endpoints}

- Between-groups absolute and relative differences in cerebral blood flow (CBF), cerebral blood volume $(\mathrm{CBV})$, mean transit time (MTT); calculated and measured $\mathrm{CMRO}_{2}$ and $\mathrm{OEF}$, respectively.

- Between-groups absolute and relative difference in MABP, arterial oxygen saturation, BIS, NIRS and subdural ICP.

\section{Data management}

All MRI and PET data are stored on a server with secured and restricted access. BIS and NIRS data are stored on an encrypted personal computer with limited access. Physiological data are recorded in a case report form (CRF). All CRFs are stored in a secure and locked area at the Department of Neuroanesthesia. Subsequently, all data are entered into a password-protected study database with secured and restricted access. Data are kept confidential.

\section{Analysis of imaging data}

MRI data

Perfusion-weighted and diffusion-weighted data are analysed as described previously. ${ }^{28-30}$

Colour-coded maps of CBF, CTH, the calculated $\mathrm{CMRO}_{2}$ and OEF for negligible tissue oxygen tension, and CBV are generated along with grey-scale apparent diffusion coefficient, and diffusion kurtosis maps are created. Regions of interests in the perilesional oedema, the contralateral normal-appearing white and grey matter, are defined on T1-weighted MRI sequences and subsequently coregistered to the perfusion and diffusion maps. Mean values in the regions of interests are calculated and correlated to the physiological parameters.

\section{PET data}

MP2RAGE will be acquired for each subject for PET coregistration. All PET images will be reconstructed with a 3D-ordered subset expectation maximization point spread function (3D-OSEM PSF) algorithm with a final isometric resolution of $2-3 \mathrm{~mm}$ and divided into time frames (twelve $5 \mathrm{~s}$ frames, six $10 \mathrm{~s}$ frames, and three $20 \mathrm{~s}$ frames for $\mathrm{CMRO}_{2}$, and $365 \mathrm{~s}$ frames, six $10 \mathrm{~s}$ frames, and three $20 \mathrm{~s}$ frames for $\mathrm{CBF}$ ).

\section{Parametric maps}

Voxel-wise parametric estimation of $\mathrm{CBF}$ and $\mathrm{CMRO}_{2}$ will be performed by using arterial blood radioactivity 
as input function and a one-tissue-compartment model based on the works of Ohta et al and Blomqvist. ${ }^{31-33}$

Oxygen extraction fraction is calculated by the formula $\mathrm{OEF}=\mathrm{CMRO}_{2} /\left(\mathrm{CBF} \cdot \mathrm{CaO}_{2}\right)$, where $\mathrm{CaO}_{2}$ is the arterial content of oxygen (haemoglobin concentration $\cdot$ oxygen saturation).

\section{Statistics}

Sample size estimation was calculated with a power analysis in Stata V.14 on a basis of earlier pilot studies with calculations of CTH, a significance level of 0.05 and a power of $0.9(\beta=0.1)$. A $10 \%$ difference in CTH was defined as a significant clinical change. In addition, a $10 \%$ difference between phenylephrine and ephedrine in cerebral oxygenation measured with NIRS was recently found clinically significant. ${ }^{916}$ No data exist on vasopressor therapy with CTH as the primary endpoint. Consequently, based on our current experience with CTH, the expected $10 \%$ difference in CTH was translated into an estimated prevasopressor MRI-CTH value of $3.216 \mathrm{~s}$ and a $10 \%$ difference, giving a mean difference of $0.3216 \mathrm{~s}$ ( $\Delta$ phenylephrine $\div$ $\Delta$ ephedrine $\geq 10 \%$ ). In both study parts a sample size of nine patients in each randomisation arm will be required. To compensate for potential dropouts and missing data, the sample size was increased by $25 \%$ to a total of 24 patients in each study part.

All data are expressed and displayed as mean and SD unless otherwise stated. The hypothesis is two-sided. Data will be analysed using conventional appropriate test statistics depending on the distribution of the individual outcome parameters. Specific tests include paired t-test or Wilcoxon signed-rank test for paired data analysis and unpaired t-test or Mann-Whitney U test for testing of between-group differences. Standard parametric or non-parametric statistical tests will be used according to the Gaussian distribution to compare MRI, PET and physiological parameters. $P$ values of less than 0.05 are considered statistically significant.

\section{Study management}

The study will be conducted in accordance with the guidelines and rules concerning quality control and quality management on clinical trials involving humans, and will follow the Good Clinical Practice and the Good Manufacturing Practice guidelines. All data regarding potential and enrolled participants will be treated confidentially. Only the investigators and relevant authorities will have access to the data. Amendments to the protocol will be communicated to the relevant authorities and the entries on the registry databases will be updated. Results will be disseminated via peer-reviewed publication and presentation at international conferences; additional summaries will be provided to patients.

\section{Ethics approval and consent to participate}

The study procedure, benefits, risks and data management will be introduced in detail to all eligible participants before they give written informed consent. Any incidents or adverse reaction will be treated immediately also postoperatively in the recovery room by the sponsor or coinvestigators, and the patient will be unblinded. Serious adverse events (SAE) or serious unexpected suspected adverse reaction (SUSAR) perioperatively or postoperatively will be recorded and reported to the Danish Health Protection Agency and the Regional Committee on Health Research and Ethics. Any deadly or life-threatening SAE that is both unexpected and suspected to be related to treatment (SUSARs) will be collected and reported to the competent authorities and relevant ethics committees in accordance with Directive 2001/20/EC, and as per national regulatory requirements as soon as possible and no later than 7 days after investigator of such an event has provided relevant knowledge. Subsequently, relevant follow-up information is to be communicated within 15 days.

\section{DISCUSSION}

In this study, we hypothesise that cerebral CTH is increased and brain oxygenation therefore reduced compared with resting $\mathrm{CMRO}_{2}$ during infusion of phenylephrine in clinically relevant doses. The study-drug dosage regimen used in this study is in contrast to other studies in which phenylephrine and ephedrine were administered as bolus doses. ${ }^{9} 1415$ In order to secure a constant exposure to the study drug and thus stable haemodynamics during the MRI and PET examinations, we found it necessary to use an infusion regimen. However, the effects on brain haemodynamics and oxygenation may be different whether the vasopressor agent is administered as a bolus dose or an infusion. For example, the gradual increase in MABP during the infusion may not elicit the same change in $\mathrm{CTH}$ and $\mathrm{CBF}$ as a bolus injection, which might elicit an autoregulatory response. ${ }^{9}$ There are methodological limitations; due to logistical and ethical considerations, we opted not to perform both MRI and PET examinations on the same subjects, although this would clearly be desirable. Accordingly, the study is divided into two different parts where patients are subjected to either MRI or PET. In addition, it is not possible to obtain simultaneous NIRS measurements during the MRI examinations due to the ferromagnetic nature of the NIRS electrode.

The study may lead to an improved understanding of the effects of commonly used vasopressors on CBF-related parameters and brain oxygenation in patients with brain pathology. The results from this study may have the potential to guide the anaesthesiologists in choosing the optimal vasopressor agent for neurosurgical procedures, which may influence surgical outcome

\section{Trial status}

The study is including patients during a period from September 2015 until December 2017. 


\section{Author affiliations}

${ }^{1}$ Department of Anesthesiology and Intensive Care-North, Section of

Neuroanesthesia, Aarhus University Hospital, Aarhus, Denmark

${ }^{2}$ Department of Neuroradiology and Center of Functionally Integrative Neuroscience,

Aarhus University Hospital, Aarhus, Denmark

${ }^{3}$ Institute of Neuroradiology, Charite Universitatsmedizin, Berlin, Germany

${ }^{4}$ Department of Nuclear Medicine and PET Center, Aarhus University Hospital,

Aarhus, Denmark

${ }^{5}$ Department of Neurosurgery, Aarhus University Hospital, Aarhus, Denmark

Acknowledgements The authors would like to thank the nurses at the Department of Neuroanesthesia, Aarhus University Hospital, and the staff at the Center of Functionally Integrative Neuroscience, Aarhus University. We would like to thank all the participating patients for their time and effort. We also thank the following people: Irene Klærke Mikkelsen (MSc, PhD), Torben Lund (Associate Professor, PhD), Dora Ziedler (neuroradiologist), Michael Geneser (neuroradiologist) and Kim Mouridsen (statistician) at Aarhus University Hospital/Aarhus University.

Contributors KUK, AT, JA and MR made substantial contribution to the conception and design of this study. KUK drafted the manuscript, with input from AT, JA, MR and LN. LN, JCHS and L $\emptyset$ were senior supervisors. GvO and NJ contributed to conduction of the clinical studies. KUK is the chief investigator and has the overall responsibility for the study. All authors participated in the preparation of, and approved for publication, the final manuscript.

Funding This work was supported by funding from Lundbeckfonden (ref R1642013-15390), Toyota Fonden (ref KJ/BG 8864 F) and the AP Moeller Foundation for the Advancement of Medical Science (ref 1718/NK PhD Vasobrain), Denmark. The study received institutional support from the Center of Functionally Integrative Neuroscience at Aarhus University and the Department of Neuroanesthesia at Aarhus University Hospital.

Competing interests None declared.

Ethics approval The study has been reported to the local GCP Unit, which is monitoring the trial. The study was approved by the Central Denmark Region Committee on Health Research Ethics (12 June 2015; 1-10-72-116-15). The trial has been approved by the Danish Health and Medicines Authority (16 June 2015, 2015042707) and the data monitoring committee Danish Data Protection Agency (2 July 2015, 1-16-02-291-15) independent from sponsor and without competing interests.

Provenance and peer review Not commissioned; externally peer reviewed.

Open Access This is an Open Access article distributed in accordance with the Creative Commons Attribution Non Commercial (CC BY-NC 4.0) license, which permits others to distribute, remix, adapt, build upon this work non-commercially, and license their derivative works on different terms, provided the original work is properly cited and the use is non-commercial. See: http://creativecommons.org/ licenses/by-nc/4.0/

(C) Article author(s) (or their employer(s) unless otherwise stated in the text of the article) 2017. All rights reserved. No commercial use is permitted unless otherwise expressly granted.

\section{REFERENCES}

1. Rasmussen M, Bundgaard $H$, Cold GE. Craniotomy for supratentorial brain tumors: risk factors for brain swelling after opening the dura mater. J Neurosurg 2004;101:621-6.

2. Sharma D, Bithal PK, Dash HH, et al. Cerebral autoregulation and $\mathrm{CO} 2$ reactivity before and after elective supratentorial tumor resection. J Neurosurg Anesthesiol 2010;22:132-7.

3. Rasmussen M, Juul N, Christensen SM, et al. Cerebral blood flow, blood volume, and mean transit time responses to propofol and indomethacin in peritumor and contralateral brain regions: perioperative perfusion-weighted magnetic resonance imaging in patients with brain tumors. Anesthesiology 2010;112:50-6.

4. Rasmussen $\mathrm{M}$, , Østergaard L, Juul N, et al. Do indomethacin and propofol cause cerebral ischemic damage? Diffusion-weighted magnetic resonance imaging in patients undergoing craniotomy for brain tumors. Anesthesiology 2004;101:872-8.

5. Petersen KD, Landsfeldt U, Cold GE, et al. Intracranial pressure and cerebral hemodynamic in patients with cerebral tumors: a randomized prospective study of patients subjected to craniotomy in propofol-fentanyl, isoflurane-fentanyl, or sevoflurane-fentanyl anesthesia. Anesthesiology 2003;98:329-36.
6. Hernández-Palazón J, Fuentes-García D, Doménech-Asensi P, et al. A comparison of equivolume, equiosmolar solutions of hypertonic saline and mannitol for brain relaxation during elective supratentorial craniotomy. Br J Neurosurg 2016;30:70-5.

7. Bratton SL, Chestnut RM, Ghajar J, et al. Guidelines for the management of severe traumatic brain injury. XIV. Hyperventilation. $J$ Neurotrauma 2007;24:S87-90.

8. Sookplung P, Siriussawakul A, Malakouti A, et al. Vasopressor use and effect on blood pressure after severe adult traumatic brain injury. Neurocrit Care 2011;15:46-54.

9. Meng L, Cannesson M, Alexander BS, et al. Effect of phenylephrine and ephedrine bolus treatment on cerebral oxygenation in anaesthetized patients. Br J Anaesth 2011;107:209-17.

10. Dubin A, Pozo MO, Casabella CA, et al. Increasing arterial blood pressure with norepinephrine does not improve microcirculatory blood flow: a prospective study. Crit Care 2009;13:R92.

11. Boerma $E C$, Ince $C$. The role of vasoactive agents in the resuscitation of microvascular perfusion and tissue oxygenation in critically ill patients. Intensive Care Med 2010;36:2004-18.

12. Sahuquillo J, Amoros S, Santos $A$, et al. Does an increase in cerebral perfusion pressure always mean a better oxygenated brain? A study in head-injured patients. Acta Neurochir Suppl 2000;76:457-62.

13. Hahn GH, Hyttel-Sorensen S, Petersen SM, et al. Cerebral effects of commonly used vasopressor-inotropes: a study in newborn piglets. PLoS One 2013;8:e63069.

14. Nissen P, Brassard P, Jørgensen TB, et al. Phenylephrine but not ephedrine reduces frontal lobe oxygenation following anesthesiainduced hypotension. Neurocrit Care 2010;12:17-23.

15. Soeding PF, Hoy S, Hoy G, et al. Effect of phenylephrine on the haemodynamic state and cerebral oxygen saturation during anaesthesia in the upright position. Br J Anaesth 2013;111:229-34.

16. Foss VT, Christensen R, Rokamp KZ, et al. Effect of phenylephrine vs. ephedrine on frontal lobe oxygenation during caesarean section with spinal anesthesia: an open label randomized controlled trial. Front Physiol 2014;5:1-9.

17. Pennekamp CW, Immink RV, Moll FL, et al. Differential effect of phenylephrine and ephedrine on cerebral haemodynamics before carotid cross-clamping during carotid endarterectomy. $\mathrm{Br} \mathrm{J}$ Anaesth 2012;109:831-3.

18. Aliane J, Dualé C, Guesmi N, et al. Compared effects on cerebral oxygenation of ephedrine vs phenylephrine to treat hypotension during carotid endarterectomy. Clin Exp Pharmacol Physiol 2017;44:739-48.

19. Sørensen H, Rasmussen P, Sato K, et al. External carotid artery flow maintains near infrared spectroscopy-determined frontal lobe oxygenation during ephedrine administration. $\mathrm{Br} J$ Anaesth 2014;113:452-8.

20. Jespersen SN, Østergaard L. The roles of cerebral blood flow, capillary transit time heterogeneity, and oxygen tension in brain oxygenation and metabolism. J Cereb Blood Flow Metab 2012;32:264-77.

21. Østergaard L, Jespersen SN, Mouridsen K, et al. The role of the cerebral capillaries in acute ischemic stroke: the extended penumbra model. J Cereb Blood Flow Metab 2013:33:635-48.

22. Østergaard L, Aamand R, Karabegovic S, et al. The role of the microcirculation in delayed cerebral ischemia and chronic degenerative changes after subarachnoid hemorrhage. J Cereb Blood Flow Metab 2013;33:1825-37.

23. Østergaard L, Engedal TS, Aamand R, et al. Capillary transit time heterogeneity and flow-metabolism coupling after traumatic brain injury. J Cereb Blood Flow Metab 2014;34:1585-98.

24. Mak PH, Campbell RC, Irwin MG.The ASA physical status classification: inter-observer consistency. American Society of Anesthesiologists. Anaesth Intensive Care 2002;30:633-40.

25. Cold GE, Tange M, Jensen TM, et al. 'Subdural' pressure measurement during craniotomy. Correlation with tactile estimation of dural tension and brain herniation after opening of dura. $\mathrm{Br} J$ Neurosurg 1996;10:69-76.

26. Saravanan S, Kocarev M, Wilson RC, et al. Equivalent dose of ephedrine and phenylephrine in the prevention of post-spinal hypotension in Caesarean section. Br J Anaesth 2006;96:95-9.

27. Doherty A, Ohashi Y, Downey K, et al. Phenylephrine infusion versus bolus regimens during cesarean delivery under spinal anesthesia. Anesth Analg 2012;115:1343-50.

28. Hansen B, Lund TE, Sangill R, et al. Experimentally and computationally fast method for estimation of a mean kurtosis. Magn Reson Med 2013;69:1754-60.

29. Ostergaard L, Sorensen AG, Kwong KK, et al. High resolution measurement of cerebral blood flow using intravascular tracer bolus passages. Part II: Experimental comparison and preliminary results. Magn Reson Med 1996;36:726-36. 
30. Mouridsen K, Hansen MB, Østergaard L, et al. Reliable estimation of capillary transit time distributions using DSC-MRI. J Cereb Blood Flow Metab 2014;34:1511-21.

31. Blomqvist $\mathrm{G}$. On the construction of functional maps in positron emission tomography. J Cereb Blood Flow Metab 1984;4:629-32.
32. Ohta S, Meyer E, Fujita $\mathrm{H}$, et al. Cerebral [150]water clearance in humans determined by PET: I. Theory and normal values. $J$ Cereb Blood Flow Metab 1996;16:765-80.

33. Ohta S, Meyer E, Thompson CJ, et al. Oxygen consumption of the living human brain measured after a single inhalation of positron emitting oxygen. J Cereb Blood Flow Metab 1992;12:179-92. 